\title{
Cartes établies au moyen d'un traceur
}

Il y a environ trois ans, le Conseil fédéral chargeait le Bureau fédéral de statistique de reprendre, tenir à jour et développer la banque de données grille d'information(1) (traduction française de "Informationsraster"), créée par l'Institut d'aménagement du territoire de 1 ' Ecole polytechnique fédérale de Zurich (ORLInstitut der ETHZ). Le passage de la grille d'information du centre de calcul de L'ETHZ (ordinateur CDC) au centre de calcul électronique de l'administration fédérale (ordinateur IBM) ne s'est pas fait sans difficultés. Dès que les nombreux travaux d'adaptation furent achevés, on envisagea la possibilité d'établir des sorties graphiques (cartes) non seulement sur 1 'imprimante rapide(1) (comme c'était déjà le cas à Zurich), mais également sur un traceur (plotter). Le centre de calcul électronique de l'administration fédérale disposant d'un traceur VERSATEC, la concrétisation de ce projet n'était donc plus qu'un affaire de temps.

1. Cartes obtenues directement par interrogation de la grille d'information (fig. 1)

Il est nécessaire de préciser d'emblée que la grille d'information est une banque de données constituée de plusieurs fichiers, l'application que nous nous proposons de décrire ici se limitant à l'un d'entre eux: le fichier des hectares. Les quelques 14 indications (on parle également de caractéristiques) stockẻes dans ce fichier se réfèrent à des carrés de $100 \mathrm{~m}$ de côté (hectares) et ont trait à la population, à l'utilisation du sol, à l'altitude, etc. A première vue, on peut s'étonner du nombre très réduit de caractéristiques enregistrées par hectare; cependant, si on se représente la superficie de la Suisse (environ 4 mio d'hectares), on peut, par simple multiplication, s'imaginer aisément le volume d'information que renferme ce fichier.

Bien qu'en pratique l'établissement de telles cartes par traceur ne présente pas de difficultés majeures, on se heurte cependant à certains problèmes lorsqu'on procède à la sélection des motifs (choix parmi une centaine de motifs). En effet, selon la caractéristique étudiēe et la région considérée, on ne peut obtenir partout des blocs distincts d'hectares de même type (décrits par le même motif). On se trouve donc fréquemment en présence d'hectares isolés et, suivant le motif choisi, il n'est pratiquement plus possible de les distinguer.

Dans notre exemple de la figure 1 , nous avons opté pour une caractéristique (altitude) se prêtant bien à ce genre de représentation et notre choix s'est portē sur un territoire (Sion (VS) et montagnes avoisinantes) dont le relief permet d'illustrer pleinement la caractéristique envisagée.

Aux neuf classes d'altitude considérées correspondent neuf motifs différents, chacun d'eux servant à représenter les hectares d'une classe donnée. Les motifs ont été choisis de telle sorte que les surfaces de faible altitude soient claires et celles de forte altitude foncées.

\section{Cartes thëmatiques de la Suisse (fig. 2)}

Ces cartes reprēsentent l'ensemble du territoire suisse, les informations qu'elles contiennent se référant à des unités supracommunales (agrégats de communes) standard, telles que cantons, districts, etc. A l'inverse de l'application présentée au paragraphe 1, l'établissement de telles cartes thématiques ne nécessite pas forcément le concours de la grille d'information. Celle-ci peut toutefois jouer un rôle important au stade de la préparation des données destinées à la représentation cartographique. Tout utilisateur ne disposant pas au préalable de données agrégées au niveau d'unités supracommunales standard a la possibilité de recourir aux services de ladite banque de données et $s^{\prime}$ évite ainsi les travaux, parfois très fastidieux, qu' exige l'élaboration de ces données.

Pour obtenir une carte du type de celle présentée à la figure 2 , il faut au moins fournir les trois informations suivantes:

a) les données se rapportant aux unités supracommunales considērées

b) la répartition désirée de ces données en un certain nombre (max. 10) de classes ou intervalles

c) les motifs devant être attribués aux différentes classes (l motif par classe)

Claude Grandjean et Klaus Arnold, Bureau fédéral de statistique, Hallwylstr. 15, 3003 Bern. 
GRAFISCHE AUSWERTUNG VOM 5.11.1977

Fig. 1

SORTIE GRAPHIQUE DU 5.11.1977

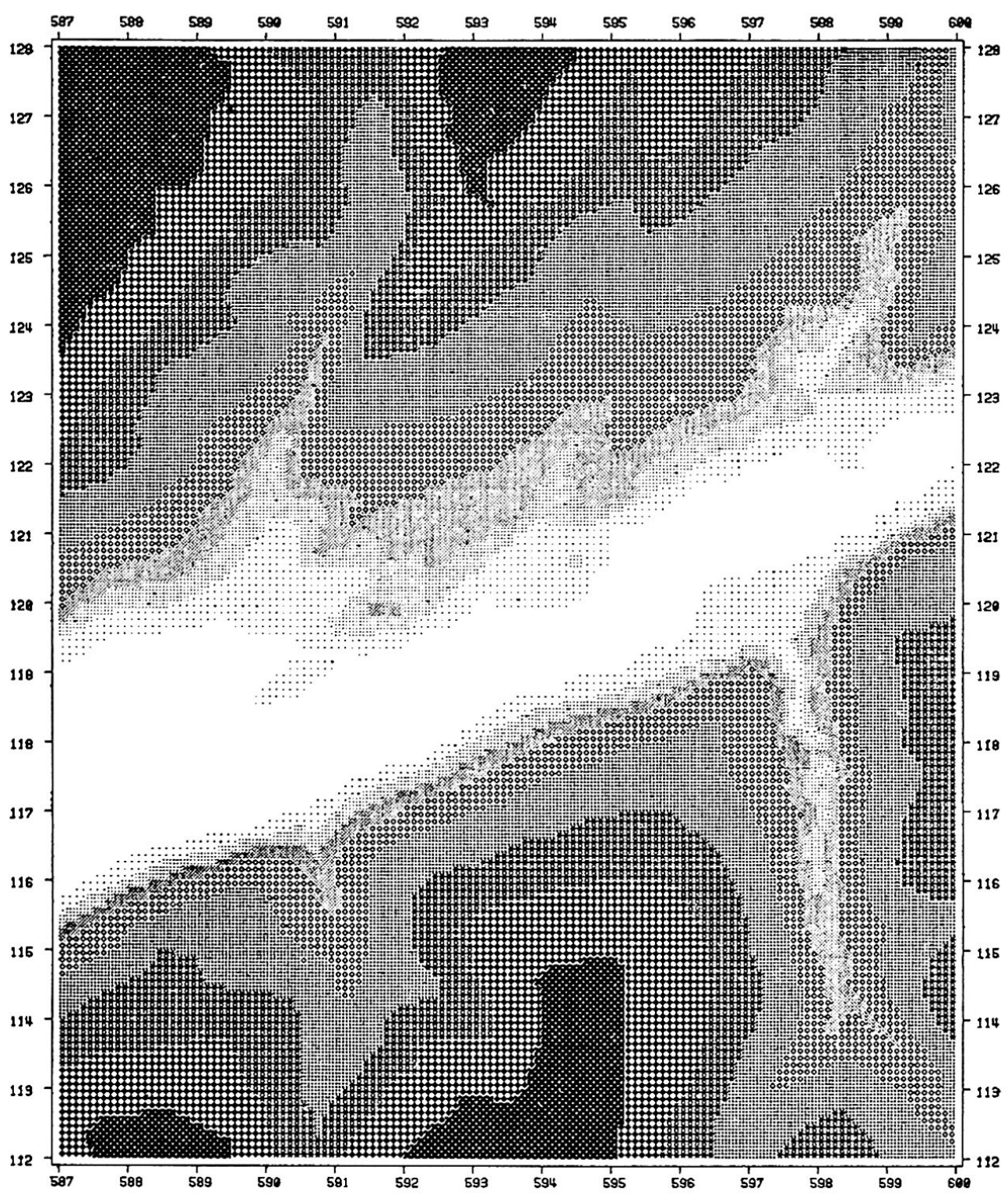

ERZ BV.BERN

DIE KOORDINATEN ENTSPRECHEN DEN KM-KOORD. DER EIDG.LANDESTOPOGRAPHIE

LES COORDONNEES CORRESPONDENT AUX COORD.KM DU SERVICE TOPOGRAPHIQUE FEDERAL

ZEICHENERKLAERUNG : STUFEN - EXPLICATION DES SIGNES: ZONES

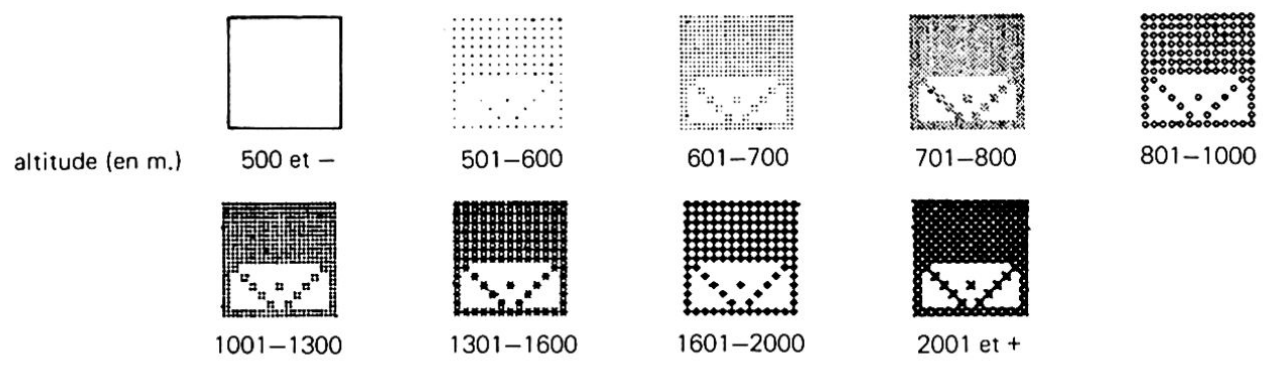




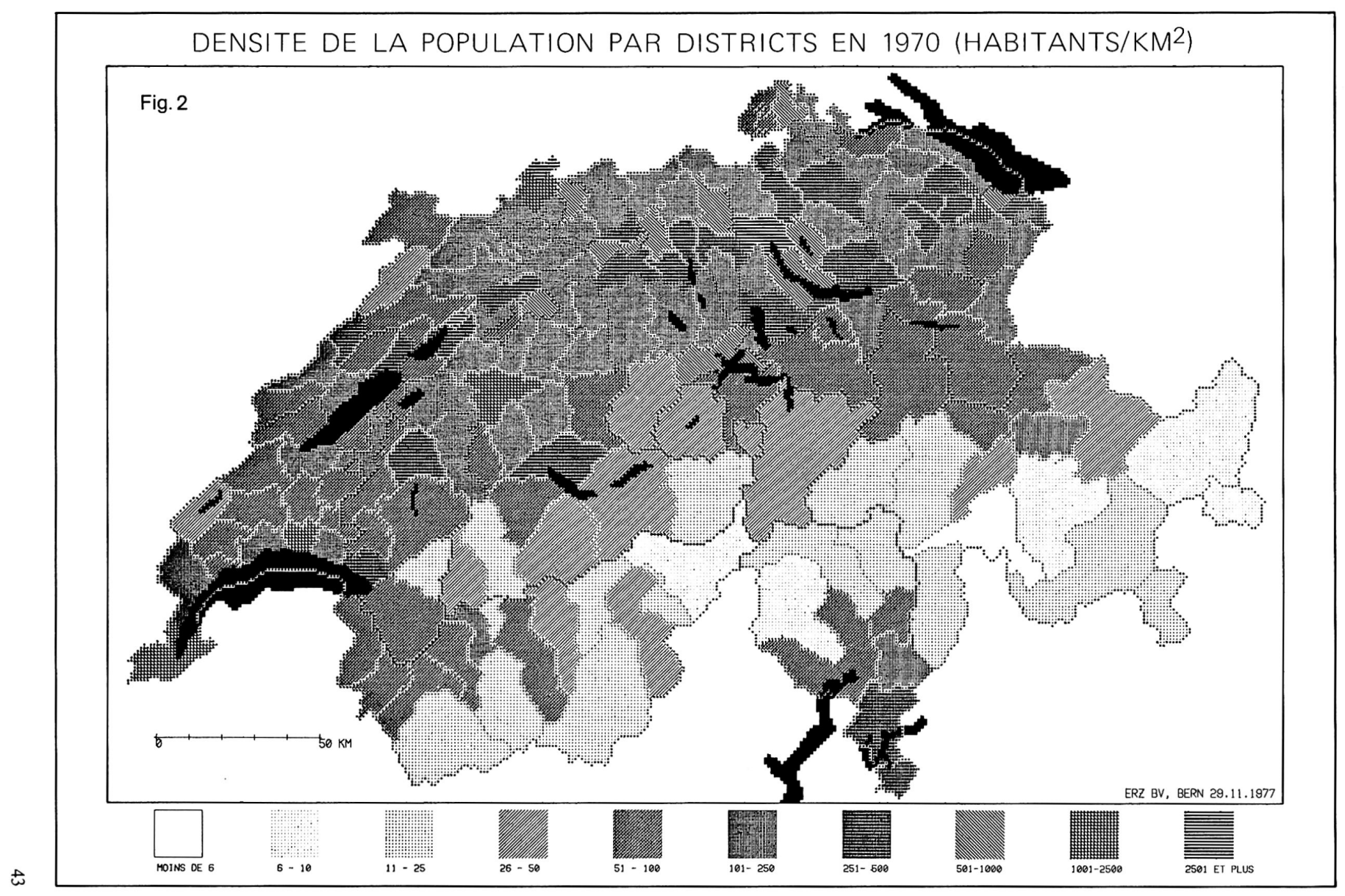


Le titre de la carte et la légende accompagnant les motifs ont pour but de décrire le contenu de la carte et l'utilisateur est libre d'en faire usage ou non.

\section{Applications spéciales}

Les exemples présentēs ci-dessus ont pu être rẻalisés grâce au langage d'interrogation de la grille d'information. Il n'est donc pas nécessaire d'être familiarisé avec le traitement électronique des données pour effectuer de telles interrogations.

Un système de banque de données doit cependant pouvoir répondre également aux besoins des utilisateurs dont les problèmes ne peuvent être résolus au moyen du langage d'interrogation. Vu que les sous-programmes d'accès aux données sont clairement séparés de la partie concernant $l$ 'interrogation proprement dite et le traitement des données (programmes d'application), une possibilité supplémentaire d'utilisation de la grille d'information est ainsi offerte aux utilisateurs connaissant le traitement èlectronique des données, lesquels n'ont pas à se soucier des questions relatives à la móthode d'accès ou à l'organisation des données. L'utilisateur peut dès lors, en se servant de ces sous-programmes, accéder à toutes les données stockées dans la banque de données, pour ensuite les exploiter au moyen d'un programme (en FORTRAN ou COBOL) qu'il aura lui-même mis au point.

Le lecteur trouvera l'exemple d'une telle application dans la présente publication, sous le titre: "Kartierung der Vegetation der Schweiz nach einem Kilometer-Raster".

'L'Institut botanique de l'Université de Berne s'est chargé lui-même du relevē des données (au km2) touchant à son domaine d'application. Quant aux indications générales, telles que frontières et lacs, elles ont été fournies par le fichier des hectares de la grille d'information. On a ensuite, à l'aide de programmes spéciaux, combiné ces deux ensembles de données, pour aboutir à la construction effective de la sortie graphique.

(1) Voir également: H. Boesch und K. Brassel: Schweizerische Landnutzungskarten, Separatabdruck aus "Geographica Helvetica", Heft 4/1973.

\section{Literaturbesprechung}

KUHLE Matthias, Beiträge zur Quartärmorphologie SE-Iranischer Hochgebirge - Die quartäre Vergletscherung des Kuh-i-Jupar, Göttinger Geographische Abhandlungen, Heft 67, Band I : Text, 209 S., Band II: 164 Abbildungen, Erich GoltzeVerlag, Göttingen 1976.

Die vorliegende Arbeit ist in der klimageschichtlich sehr wesentlichen Auseinandersetzung zwischen einigen französischen und deutschen Arbeiten um die Grösse und Höhenlage der eiszeitlichen Vergletscherung der iranischen Hochgebirge zu sehen. Kuhle hat die über $4000 \mathrm{~m}$ aufragenden Massive bei Kerman sehr detailliert untersucht und kommt zu folgenden Schlüssen: Eine ältere Vereisung (Riss) mit einer Schneegrenze um $2960 \mathrm{~m}$ erzeugte eine gewaltige Gebirgsvergletscherung mit über $20 \mathrm{~km}$ breiten Eisloben im Vorland und eine tiefste, durch Moränen belegte Eisrandlage auf 1900m Höhe. Eine jüngere Vereisung (Würm) mit einer Schneegrenze um $3060 \mathrm{~m}$ war räumlich etwas beschränkter, aber immer noch durch Glet- scherlängen von $10 \mathrm{~km}$ und Endlagen zwischen $2160 \mathrm{~m}$ und $2500 \mathrm{~m}$ gekennzeichnet.

Mit diesen Befunden, reich dokumentiert mit 164 Abbildungen, setzt sich Kuhle in Widerspruch zu zahlreichen bisherigen Arbeiten in anderen Gebirgsräumen Irans, stimmt aber überein mit jüngeren Arbeiten im zentraliranischen Shir-KuhMassiv.

Zu denken gibt uns bloss der bedeutende Unterschied zwischen älterer und jüngerer Vereisung bei einer bloss um 100m verschiedenen Schneegrenzdepression, vor allem die gewaltige Vorlandvergletscherung der älteren Eiszeit in diesem kontinentalen Raum auf rund $30^{\circ} \mathrm{n}$. Br.. Wir wären wohl dankbar, wenn diese klimageschichtlich sehr wesentlichen Befunde in Zukunft durch flankierende Untersuchungen (z.B. absolute Datierungen, Paläoböden, klimatologische und zirkulationsmechanische Interpretationen) untermauert würden.

GH $1 / 1978$

B. Messerli 